\title{
Involuntary Motor Activity in Pianists Evoked by Music Perception
}

\author{
Jens Haueisen $^{1}$ and Thomas R. Knösche ${ }^{2}$
}

\begin{abstract}
Pianists often report that pure listening to a well-trained piece of music can involuntarily trigger the respective finger movements. We designed a magnetoencephalography (MEG) experiment to compare the motor activation in pianists and nonpianists while listening to piano pieces. For pianists, we found a statistically significant increase of activity above the region of the contralateral motor cortex. Brain surface
\end{abstract}

\section{INTRODUCTION}

Musicians learn and perform quite complex trains of movement. They frequently report that listening to a well-trained piece of music can trigger the associated movements (e.g., finger movements in pianists). This suggests a strong coupling between perception and production of music and leads to a number of questions:

1. Is there also motor cortex activation without actual movements?

2 . Is this activation involuntary?

3. Which parts of the motor system are active? Is the localization of the activity similar to the one found in motor imagery studies?

The question of the relationship between perception and production of well-trained music in piano learners has been investigated by Bangert, Parlitz, and Altenmüller (1999) using electroencephalography (EEG). They found that the spatial EEG patterns become increasingly similar between the perception and production task when the subjects proceed in their learning. This suggests some sort of coupling between music perception and motor activity in musically trained individuals. However, it is not clear which part of the motor system is activated and if this activation is involuntary.

Motor imagery can be seen as a voluntary activation of the motor system without actual movement. Functional magnetic resonance imaging (fMRI) revealed the participation of the primary motor cortex (M1), the supple-

\footnotetext{
${ }^{1}$ Friedrich-Schiller-University, Jena, Germany, ${ }^{2}$ Max-PlanckInstitute of Cognitive Neuroscience, Leipzig, Germany
}

current density (BSCD) reconstructions revealed a spatial dissociation of this activity between notes preferably played by the thumb and the little finger according to the motor homunculus. Hence, we could demonstrate that pianists, when listening to well-trained piano music, exhibit involuntary motor activity involving the contralateral primary motor cortex (M1).

mentary motor area (SMA), and the premotor cortex (PMC) (Lotze et al., 1999; Porro et al., 1996; Roth et al., 1996). Stefan et al. (1995) used positron emission tomography (PET) to investigate imagery of joystick movements. They found activity in, for example, the PMC, anterior cingulate cortex, and parietal cortex. The participation of the M1 in imagery of finger movements was revealed by Schnitzler, Salenius, Salmelin, Jousmaki, and Hari (1997) using magnetoencephalography (MEG). Finally, a number of EEG studies suggest activity in primary sensorimotor cortex due to imagery of hand movements (McFarland, Miner, Vaughan, \& Wolpaw, 2000; Pfurtscheller, Neuper, Ramoser, \& Muller-Gerking, 1999; Pfurtscheller \& Neuper, 1997; Beisteiner, Hollinger, Lindinger, Lang, \& Berthoz, 1995).

These studies imply that, if motor cortex activation caused by the perception of music is similar to activation due to motor imagery, we should detect at least involvement of the M1, possibly also the SMA, PMC, anterior cingulate, and parietal cortex. We designed an MEG experiment to compare the motor activation related to musical stimuli in pianists and nonpianists. In order to focus on involuntary activation of the motor cortex we chose a task that was completely unrelated to movements. If there was indeed an involuntary motor activation, we would have expected increased activity in the motor areas in pianists, but not in nonpianists. Additionally, for activity originating from the M1, a spatial dissociation between the activity related to notes played with different fingers had to be expected.

MEG is one of the methods that provides sufficient spatial resolution, allowing separation of different types of motor activities (e.g., M1, SMA, PMC). 


\section{RESULTS}

In order to test the hypothesis whether or not there is motor activity between -300 and 0 msec before the notes' onsets we employed two different methods to localize the brain activity. First, we used the MEG derivative method to test for differential activity in various brain regions. Figure $1 \mathrm{a}-\mathrm{c}$ summarizes the re- sults as relative differences between pianists and nonpianists, integrated over the pretrigger period. The difference plot clearly shows a focus of activity above the left central sulcus, whereas other areas exhibit little differential activation. Statistical tests have been carried out for five regions above the auditory and motor cortex (Figure 1d). Only the region including the focus above the left rolandic area yielded significant differences

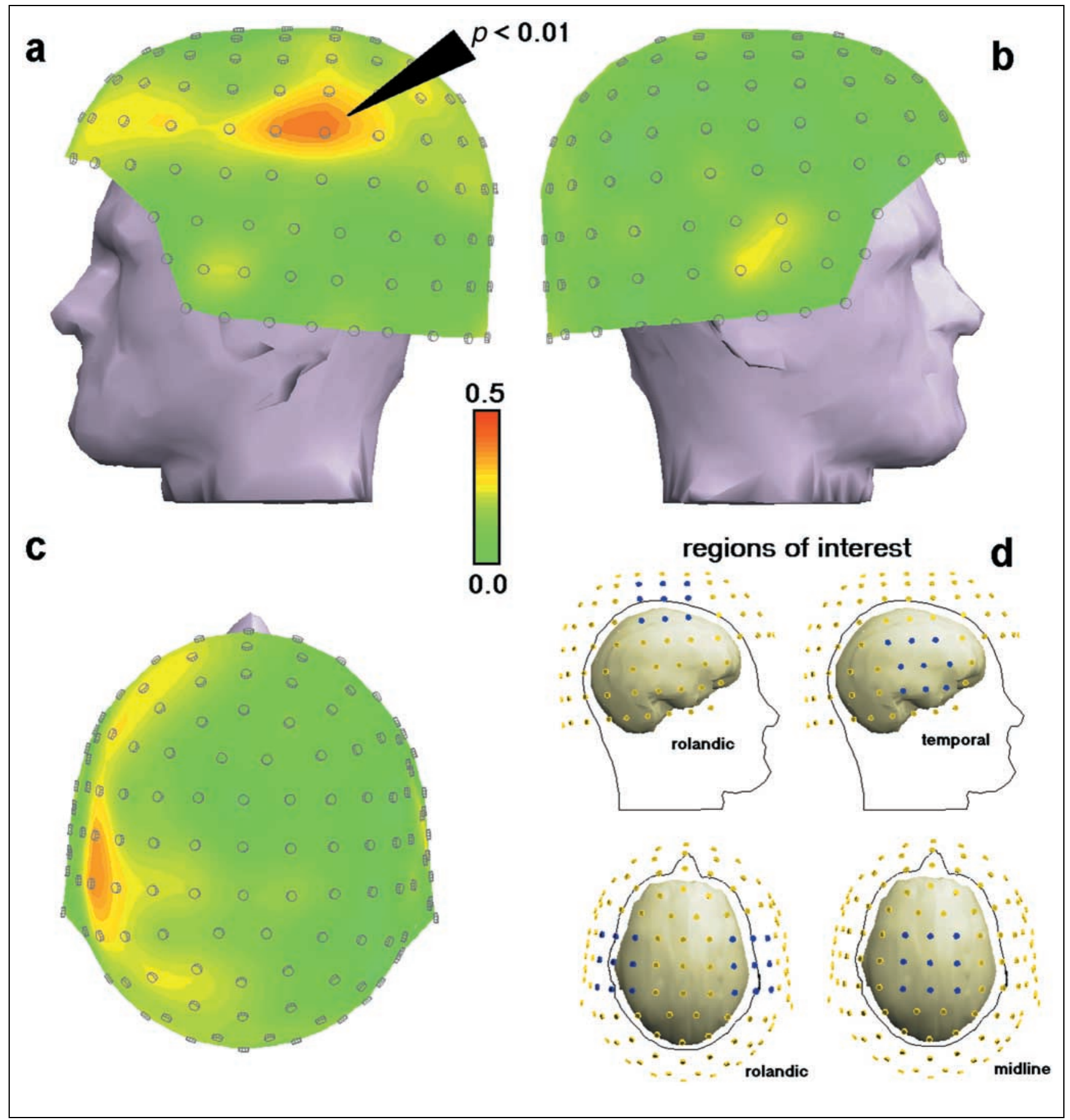

Figure 1. Summary of MEG derivative results. The plots $(\mathrm{a}-\mathrm{c})$ show the relative differences of the MEG derivatives between pianists and nonpianists group averages. The plotted measure is the magnitude of the surface gradient of the MEG. The channels that were used for the regions of interest of the statistical test are indicated in (d). 
between pianists and nonpianists $(p<.01)$. The location of the MEG derivative focus suggested the involvement of the M1 in pianists.

Second, we applied the brain surface current density (BSCD) method to separate averages of the notes preferably played by the thumb and the little finger. The differences between pianists and nonpianists are depicted in Figure 2. There is a clear dissociation between the localizations of thumb and little finger, being separated about $8 \mathrm{~mm}$ in the inferior-superior direction $(p=.05$, multivariate test: thumb and little finger; inferior and superior region; source strength). This is in agreement with the motor homunculus and confirms the involvement of the M1. Also, the Talairach coordinates (thumb: F c 3; little finger: E3 c 2), are in agreement with literature values for the hand area of the primary motor area (e.g., E-F b-c 3 in a PET study by Carey, Abbot, Egan, Tochon-Danguy, \& Donnan, 2000). We found additional activity in the left temporal region for both conditions. In the little finger condition, the right occipital lobe was active, too.

\section{DISCUSSION}

The aim of this study was to answer the question whether the mere perception of well-trained piano music can involuntarily evoke motor cortex activity in pianists without actual movement. Our results show that this is indeed the case. Furthermore, we provide strong evidence that primary motor activity is involved in this process.

From the results of Bangert et al. (1999) it is not clear which brain regions are additionally active during music perception in piano players. Studies on motor imagery using electrophysiological methods (EEG, MEG) mainly suggest involvement of the primary motor or sensorimotor cortex (McFarland et al., 2000; Pfurtscheller \& Neuper, 1997; Pfurtscheller et al., 1999; Schnitzler et al., 1997; Beisteiner et al., 1995). Methods that monitor cerebral blood flow, like fMRI and PET, also found involvement of the SMA, PMC, anterior cingulate cortex, and parietal cortex (Lotze et al., 1999; Porro et al., 1996; Roth et al., 1996; Stefan et al., 1995).

In agreement with the above-mentioned electrophysiological studies, our results suggest the predominant activation of the M1. We did not detect any activity arising from other constituents of the motor system that are known for their contribution to motor programming (like SMA or PMC). However, some sort of motor programming must have taken place because we detected M1 activity before the onset of the notes, and the finger dissociation indicated that the subjects knew with which finger the next note would normally be played. We speculate that, because of the high level of skill of our subjects, the motor programming processes were highly automated and required little recruitment in the motor programming areas. Another possible explanation for a

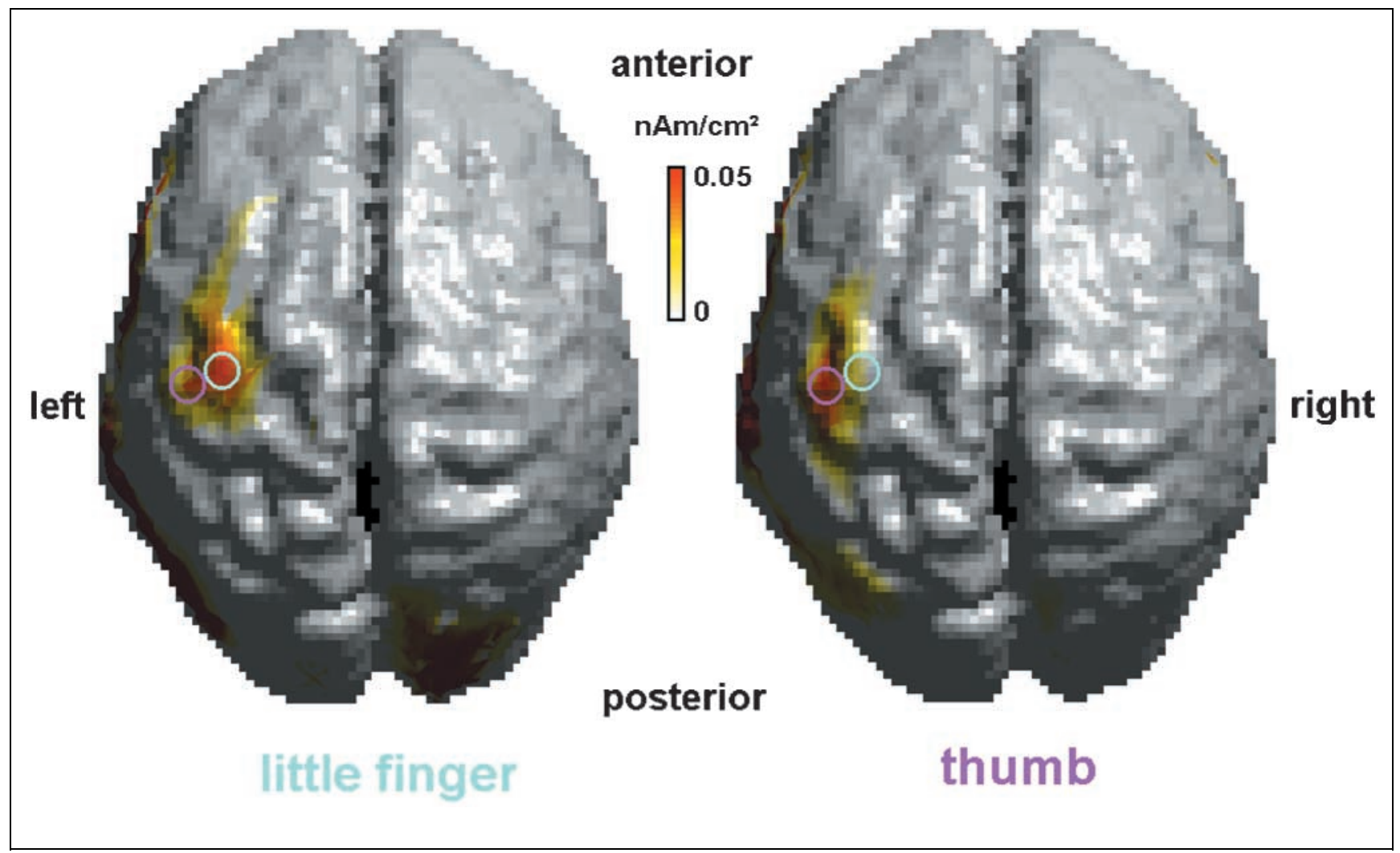

Figure 2. BSCD mapping results. The plots show the differences of the current source density between pianists and nonpianists group averages plotted on the surface of a standard MRI. 
missing SMA contribution can be found in the local anatomy of the cortex. Lang et al. (1991) and Cheyne and Weinberg (1989) explain the failure of MEG to detect SMA as a possible generator of the early readiness potential prior to voluntary movements by the fact that several studies in primates (using single-cell recordings) and humans (using PET) have revealed that the SMA is bilaterally active even in unilateral movements. The activity of the SMA in both hemispheres can be described by two tangential dipoles that are antiparallel to each other and therefore do not produce an external magnetic field. This could also explain that no SMA has been found in a number of motor imagery studies using EEG or MEG (McFarland et al., 2000; Pfurtscheller \& Neuper, 1997; Pfurtscheller et al., 1999; Schnitzler et al., 1997; Beisteiner et al., 1995).

The activation of motor areas by the perception of associated feedback has been demonstrated in other fields as well; for example, evidence has been shown that pure visual perception of movements also activates the associated motor areas (Cochin, Barthelemy, Roux, \& Martineau, 1999; Hari et al., 1998).

The left temporal activity (Figure 2) that was different between pianists and nonpianists (not significant in this study) could be a hint to some additional auditory processing in pianists. Interestingly, there is also differential activity in the right occipital cortex in the little finger condition only. This seems not readily explainable, although Zatorre, Evans, and Meyer (1994) reported increased $\mathrm{rCBF}$ in this region during the perception of music.

Note that the participants of this experiment were advanced students, not professional pianists. Thus, even more pronounced effects could be expected in professionals. One problem in this type of study is the inevitable overlap of the activity related to the notes within one piece. In order to diminish this problem we had to choose rather simple representations of the music using a single voice only. This of course affected the emotional content of the music.

In conclusion, our results strongly support the idea of a close coupling between perception and execution systems in the brain. In particular, we have provided evidence that M1 activity can be evoked by and synchronized to the associated perceptive feedback.

\section{METHODS}

\section{Subjects}

The study was carried out on 26 subjects, 6 of which had to be excluded due to problems involving head position measurement. Of the remaining 20 participants, 10 had a history of piano playing of at least 7 years ( 6 women; age $21.4 \pm 3.1$; all right-handed). The other 10 subjects (all women; age $21.9 \pm 2.8$; one left-handed) had a comparable experience in producing music (11.9 \pm 4.0 vs. 13.2 \pm 2.3 years as pianists). All of the nonpianists were singers in a university chorus. Some of them had played an instrument other than the piano in the past (3 strings, 5 flute) for $4.8 \pm 4.2$ years. All subjects gave written informed consent to participate in the study. The study was approved by the ethics committee.

\section{Material}

The stimulus material consisted of 24 sequences of well-known piano pieces, generated on a MIDI-based synthesizer. All pieces were typical piano pieces without singing text or singing tradition and were played on a piano in order to reduce the possibility of undesired silent singing. For each of the pieces, a second version existed, with one note out of key (roughly in the middle of the piece). These pieces were used as filler items. All pieces were played in a single voice (melody only, no bass) and contained between 18 and 64 notes of the principal theme (between 8 and 29 sec; average $14.9 \mathrm{sec}$; standard deviation $4.6 \mathrm{sec}$ ). They where presented in a randomized sequence of 192 items with 3-sec interstimulus interval. Each of the 48 pieces (24 correct and 24 incorrect) occurred exactly four times. The resulting 192 stimuli were divided into four block with several minutes break in between. Their sequence was balanced by presenting them in inverse order to half of the subjects in each group. The notes of the presented pieces were shown to the subjects before the experiment. Based on these notes, each pianist rated each piece $(10$ pianists $\times 24$ pieces $)$. The rating question was: How well do you perform this piece? The scale included perfect, very well, well, poor, and very poor. The overall rating was $7 \%$ perfect, $19 \%$ very good, 66\% good, $5 \%$ poor, and $2 \%$ very poor. A similar rating, the familiarity, was obtained from the nonpianists. The familiarity was high in 35\%, quite good in $23 \%$, somewhat in $13 \%$, little in $11 \%$, and very little or not at all in $17 \%$.

The volume was adjusted to $45 \mathrm{~dB}$ above the individual hearing threshold at $1 \mathrm{kHz}$ (separately for left and right ear).

\section{Task}

Because this study focused on the involuntary activation of motor areas, the task for the subjects had to be unrelated. They were instructed to detect a certain piece of music and press a button (with the thumb of their dominant hand) when this piece contained a wrong note. The response button was held by the dominant hand during the entire experiment, the thumb resting on the button and the hand resting on the thigh. They were further asked to relax, keep their heads and eyes still, and refrain from blinking as much as possible. The task was accomplished correctly by all subjects. 


\section{Recording}

The measurements were performed with the subjects sitting with their eyes open in a magnetically shielded room. A total of 148 channels of MEG (MAGNES II, 4-D Neuroimaging, San Diego, CA), 2 channels of EOG (horizontal and vertical, bipolar), and 2 channels of EMG (bipolar) were recorded. The EMG was measured to make sure that no real movements were carried out (bipolar over both extensor and flexor digitorum communis muscles). EOG was used to detect eye movements. The sampling frequency was $506.7 \mathrm{~Hz}$ (band pass 0.1 to $100 \mathrm{~Hz}$ ). The position of the head with respect to the dewar was determined before and after each block.

\section{Data Preprocessing}

In order to minimize superposition of the fields evoked by different notes, the trigger points were selected at the onsets of notes separated by at least
$500 \mathrm{msec}$ from both the previous and the following note. These notes were approximately equally distributed over the entire lengths of the pieces. Furthermore, no trials containing eye blink or movement artifacts and all trials associated to stimuli containing wrong notes were used. This yielded about 225 sweeps per block average. Two additional sets of averages were computed by taking into account only those notes that are preferably played by the little finger or the thumb, respectively. The rating was done by a professional musician. An example of the rating is given in Figure 3. This yielded about 30 sweeps per block and finger condition.

Subsequently, the averages of the four blocks belonging to one subject were transformed to an average sensor position using a source space projection scheme (Numminen, Ahlfors, Ilmoniemi, Montonen, \& Nenonen, 1995) and averaged. The resulting subject averages were transformed again to the average sensor position of all subjects within each group (pianists or nonpian-
Figure 3. Scores of 3 out of 24 melodies presented: (a) Mozart, (b) Bach, (c) Schumann. The numbers above the notes indicate the fingers that would be commonly used to play this note (1-thumb, 5-little finger).
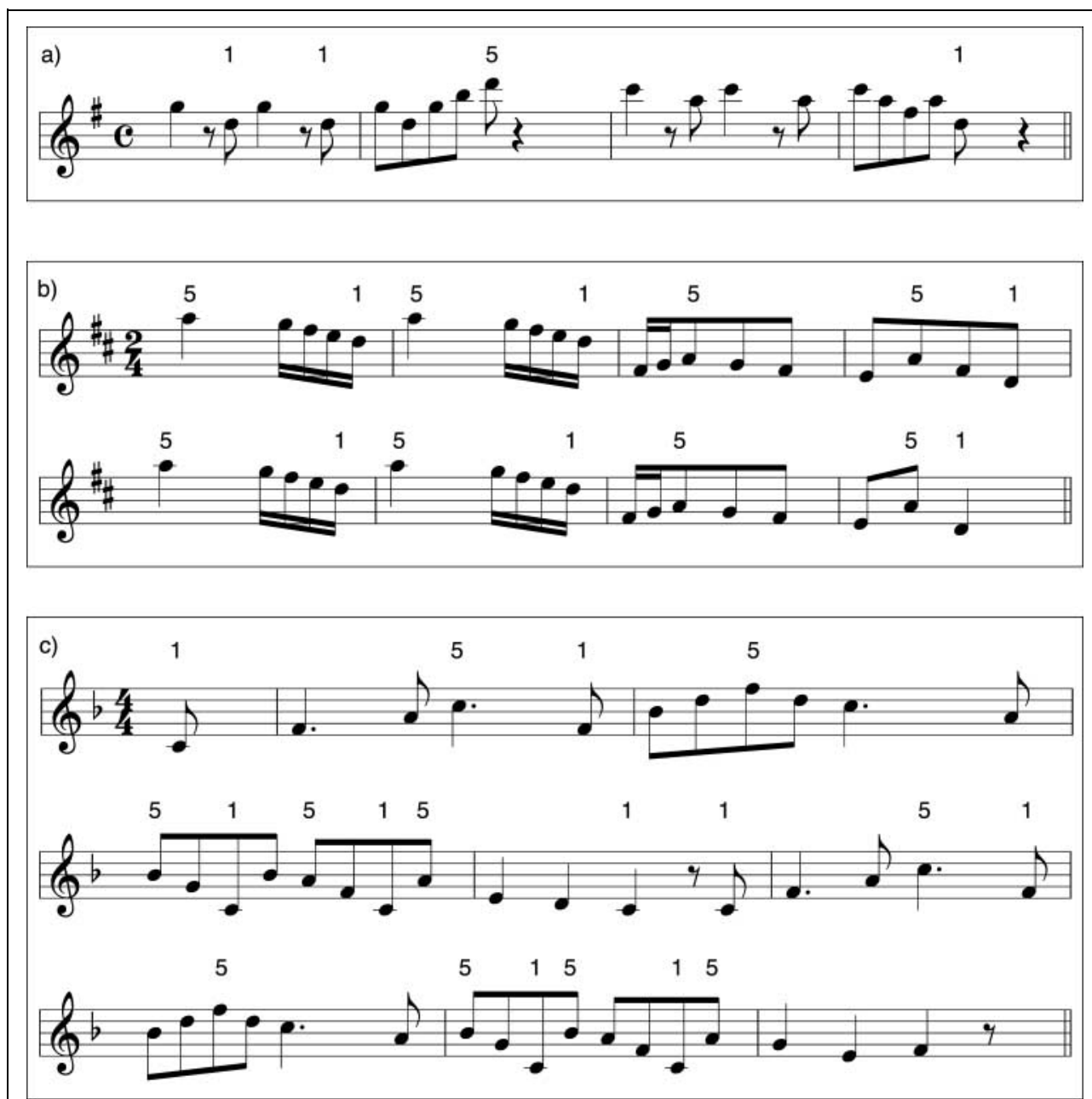
ists). This two-stage procedure proved more stable than the immediate transformation of the blocks to the global standard position. Now, for each subject, an average was available as if measured at the same sensor position, rendering the computation of grand averages and statistics possible.

Due to the large drifts in the MEG signals it was necessary to perform a baseline correction per sweep. Because motor activity is to be expected from several hundred milliseconds before to several hundred milliseconds after the onset of the notes, there is no signalfree stretch of data. This makes it inevitable that the motor activity is diminished by the baseline correction. In order to gain the maximum statistical power, the baseline was chosen to cover the strongest nonmotor activity, namely, the primary auditory components (20.250 msec). Note that primary auditory components mainly consist of higher frequencies, which are not projected into the analysis interval by a baseline of 230-msec length. We have to be aware, however, that part of the (slow) motor activity found in the analysis interval might originate from the baseline interval.

\section{Data Analysis}

\section{MEG Derivative}

Recordings with magnetometers are typically composed of two-lobed dipolar patterns with the zero line directly above the active brain region. For assessment and statistical analysis, it is useful to have a one-to-one correlation between active brain areas and activation peaks on the sensor array. Therefore, we used the norm of the tangential derivative of the radial component of the magnetic field.

$$
M(\vec{r})=\mid\left(\frac{\partial B(\vec{r})}{\partial \vec{u}(\vec{r})}\right)^{2}+\left(\frac{\partial B(\vec{r})}{\partial \vec{v}(\vec{r})}\right)^{2}
$$

The measure $M$ at the sensor position $\vec{r}$ is the sum of the squares of the partial derivatives of the measured (radial) component of the magnetic field $B$ with respect to the two (mutually orthogonal) tangential directions $\vec{u}$ and $\vec{v}$.

This measure exhibits maxima directly above the foci of the brain activity (Hari, 1993). For analysis, we chose the time interval where the least contamination by auditory-evoked and event-related activity is to be expected, that is, -300 to 0 msec.

We computed the grand average over all subjects and all time samples of the analysis interval. According to the activity distribution and the anatomical structure of the underlying brain, 5 regions of interest were defined, containing 9 channels each: left/right temporal, left/right rolandic, midline rolandic. For each of these regions, the data were integrated over channels and time $(-300 \ldots 0$ msec) for each subject. A two-tailed Student $t$ test was computed for each region of interest between the two groups. In order to ensure that the data were Gauss distributed, they were subjected to a square root transform and then assessed for normality using the Kolmogorov-Smirnov test.

\section{BSCD Mapping}

In order to reveal finer details about the investigated brain activity, the localization of the sources of the MEG was performed. Because the targeted activity in the M1 is expected to suffer a great deal of both spatial and temporal overlap by other activity, we chose a localization scheme that does not require any a priori knowledge on the nature of the generators. This method, referred to as BSCD mapping (Knösche, Praamstra, Stegeman, \& Peters, 1996), reconstructs tangential currents on the surface on the standard brain model.

This standard brain has been obtained by averaging a number of Talairach-scaled MRI scans. Then, the brain surface was extracted and triangulated (about 1100 triangles). The resulting model was used to account for the effects of volume current within the boundary element scheme, as well as to define the brain surface where the tangential currents were reconstructed. For every individual, the sensor array was linearly scaled in order to preserve the principal distances between the head surface and the sensors and therefore to account for different head sizes and shapes. Nasion, left and right ear, and $\mathrm{Cz}$ anatomical landmarks of the individual head (originating from the digitizer, Isotrak II, Polhemus, Colchester, VT) were projected onto the surface of the standard head model and the sensor array was scaled by the distances between projected and original landmarks.

The data analysis was performed using ASA (A.N.T. Software, Enschede, Netherlands).

\section{Acknowledgments}

The authors thank A. D. Friederici, G. Curio, T. Weiß, and T. Rosburg for fruitful discussions and B. Maeß and U. Oertel for essential help in carrying out the experiments.

Reprint requests should be sent to Jens Haueisen, Biomagnetisches, Zentrum, Universität Jena, Philosophenweg 3, 07743 Jena, Germany, or via e-mail: haueisen@biomag.uni-jena.de.

\section{REFERENCES}

Bangert, M., Parlitz, D., \& Altenmüller, E. (1999). An interface for complex auditory-sensorimotor integration: Where the pianist's cortex maps perception to action. Neuroimage, 9, S419.

Beisteiner, R., Hollinger, P., Lindinger, G., Lang, W., \& Berthoz, A. (1995). Mental representation of movements. Brain potentials associated with imagination of hand movements. Experimental Brain Research, 78, 604-612.

Carey, L. M., Abbot, D. F., Egan, G. F., Tochon-Danguy, H. J., \& 
Donnan, G. A. (2000). The functional neuroanatomy and long-term reproducibility of brain activation associated with a simple finger tapping task in older healthy volunteers: A serial PET study. Neuroimage, 11, 124-144.

Cheyne, D., \& Weinberg, H. (1989). Neuromagnetic fields accompanying unilateral finger movements: Pre-movement and movement-evoked fields. Journal of Cognitive Neuroscience, 11, 491-501.

Cochin, S., Barthelemy, C., Roux, S., \& Martineau, J. (1999). Observation and execution of movement: Similarities demonstrated by quantified electroencephalography. European Journal of Neuroscience, 11, 1839-1842.

Hari, R. (1993). Magnetoencephalography as a tool of clinical neurophysiology. In E. Niedermeyer \& F. Lopes da Silva (Eds.), Electroencephalography: Basic principles, clinical applications, and related fields (pp. 1035-1065). Baltimore: Williams and Wilkins.

Hari, R., Forss, N., Avikainen, S., Kirveskari, E., Salenius, S., \& Rizzolatti, G. (1998). Activation of human primary motor cortex during action observation: A neuromagnetic study. Proceedings of the National Academy of Sciences, U.S.A., 95, 15061-15065.

Knösche, T. R., Praamstra, P., Stegeman, D., \& Peters, M. J. (1996). Linear estimation discriminates midline sources and a motor cortex contribution to the readiness potential. Electroencephalography and Clinical Neurophysiology, 99, 183-190.

Lang, W., Cheyne, D., Kristeva, R., Beisteiner, R., Lindinger, G., \& Deecke, L. (1991). Three-dimensional localization of SMA activity preceding voluntary movement. A study of electric and magnetic fields in a patient with infarction of the right supplementary motor area. Experimental Brain Research, 87, 688-695.

Lotze, M., Montoya, P., Erb, M., Hulsmann, E., Flor, H., Klose, U., Birbaumer, N., \& Grodd, W. (1999). Activation of cortical and cerebellar motor areas during executed and imagined hand movements: An fMRI study. Journal of Cognitive Neuroscience, 11, 491-501.
McFarland, D. J., Miner, L. A., Vaughan, T. M., \& Wolpaw, J. R. (2000). Mu and beta rhythm topographies during motor imagery and actual movements. Brain Topography, 12, 177-186.

Numminen, J., Ahlfors, S., Ilmoniemi, R., Montonen, J., \& Nenonen, J. (1995). Transformation of multichannel magnetocardiographic signals to standard grid form. IEEE Transactions on Biomedical Engineering, 42, 72-78.

Pfurtscheller, G., \& Neuper, C. (1997). Motor imagery activates primary sensorimotor area in humans. Neuroscience Letters, 239, 65-68.

Pfurtscheller, G., Neuper, C., Ramoser, H., \& Muller-Gerking, J. (1999). Visually guided motor imagery activates sensorimotor areas in humans. Neuroscience Letters, 269, 153-156.

Porro, C. A., Francescato, M. P., Cettolo, V., Diamond, M. E., Baraldi, P., Zuiani, C., Bazzocchi, M., \& di Prampero, P. E. (1996). Primary motor and sensory cortex activation during motor performance and motor imagery: A functional magnetic resonance imaging study. Journal of Neuroscience, 16, 7688-7698.

Roth, M., Decety, J., Raybaudi, M., Massarelli, R., Delon-Martin, C., Segebarth, C., Morand, S., Gemignani, A., Decorps, M., \& Jeannerod, M. (1996). Possible involvement of primary motor cortex in mentally simulated movement: A functional magnetic resonance imaging study. NeuroReport, 7 , 1280-1284.

Schnitzler, A., Salenius, S., Salmelin, R., Jousmaki, V., \& Hari, R. (1997). Involvement of primary motor cortex in motor imagery: A neuromagnetic study. Neuroimage, 6, 201-208.

Stefan, K. M., Fink, G. R., Passingham, R. E., Silbersweig, D., Ceballos-Baumann, A. O., Frith, C. D., \& Frackowiak, R. S. (1995). Functional anatomy of the mental representation of upper extremity movements in healthy subjects. Journal of Neurophysiology, 73, 373-386.

Zatorre, R. J., Evans, A. C., \& Meyer, E. (1994). Neuronal mechanisms underlying melodic perception and memory for pitch. Journal of Neuroscience, 14, 1908-1919. 